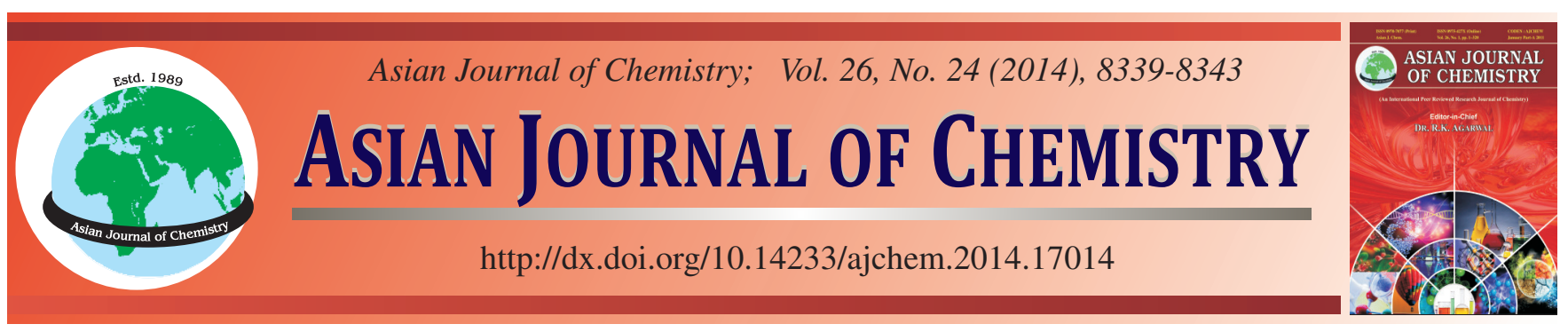

\title{
Determination and Quantitative Analysis of Fatty Acids from Hippophae rhamnoides L. Using Reversed-Phase HPLC with Fluorescence Detection and Atmospheric Chemical Ionization-Mass Spectrometry
}

\author{
Na Hu ${ }^{1,2,3}$, Zhongyin JI ${ }^{1,3}$, Ainong Wang ${ }^{1,3}$, Lijuan Han ${ }^{1,3}$, Jinmao You $^{1}$ and Yourui Suo ${ }^{1,2, *}$
}

${ }^{1}$ Key Laboratory of Tibetan Medicine Research, Northwest Institute of Plateau Biology, Chinese Academy of Sciences, Xining 810001, P.R. China ${ }^{2}$ Academy of Agriculture and Forestry, Qinghai University, Xining 810016, P.R. China

${ }^{3}$ University of the Chinese Academy of Sciences, Beijing 100049, P.R. China

*Corresponding author: Tel/Fax: +86 971 6143857; E-mail: yrsuo@nwipb.cas.cn

\begin{abstract}
In the present study, a selective and sensitive method using 2-(11H-benzo[a]carbazol-11-yl) ethyl 4-methylbenzenesulfonate as labeling reagent based on pre-column derivatization for the quantitative analysis of fatty acids was developed and validated. The results indicated the proposed method exhibited excellent selectivity, sensitivity, reproducibility and applicability. Good linear correlations were obtained for each fatty acid with correlation coefficients greater than 0.9994 . The values of LOQs and LODs ranged from 1.34 to $6.64 \mathrm{ng} \mathrm{mL}^{-1}$ and 0.42 to $1.82 \mathrm{ng} \mathrm{mL}^{-1}$, respectively. This method was successfully employed to analyze fatty acid composition in the seeds of Hippophae rhamnoides $L$. The results showed that seeds of Hippophae rhamnoides $L$. seeds are rich in fatty acids and the contents of unsaturated fatty acids are much higher than that of saturated fatty acids. In addition, the contents of total fatty acids, unsaturated fatty acids and each fatty acid are various among different origins. The analysis of fatty acids in seeds of Hippophae rhamnoides L. is important to the pharmacological research of Hippophae rhamnoides $L$.
\end{abstract}

Keywords: Fatty Acids, Hippophae rhamnoides L., Pre-column fluorescence labelling, HPLC-FLD, APCI-MS.

\section{INTRODUCTION}

Hippophae rhamnoides L. (Sea buckthorn, SBT) belongs to the genes of Hippophae L. in Elaeagnaceae family, which is extensively distributed over Europe and Asia ${ }^{1}$. It is a hardy plant, which is useful for land reclamation and farmstead protection for its vigorous vegetative reproduction and complex root system ${ }^{2}$. In China, the main distribution area is the northwest regions, especially for the Qaidam Basin of QinghaiTibet platuau. Hippophae rhamnoides $L$. has been recorded in Tibetan medical classics "Jing Zhu Ben Cao" and "Si Bu Yi Dian" as the traditional medicine material and used for centuries as a traditional herbal medicine for the treatment of asthma ${ }^{3}$, skin diseases, anti-stress ${ }^{4}$, anti-atherogenic ${ }^{5}$, lung disorders, gastric ulcers ${ }^{6}$, diminishing inflammation ${ }^{7}$ and tumors ${ }^{8}$. All parts of this plant are considered to be a good source of large number of bioactive ingredients such as tocopherols, sterols, carotenoids ${ }^{9}$, ascorbic acid ${ }^{10}$, flavonoids ${ }^{11}$, etc. Recently, the nutritional values of Hippophae rhamnoides $L$. berries have attracted more attentions in North America and Europe. Berry products of Hippophae rhamnoides L. such as fruit wine, fruit juice, fruit power, etc. are popular foods in the United States, Canada, Finland, Germany and some other European countries. Seeds of Hippophae rhamnoides L., rich in fatty acids, are the by-products in the processing of berry production, which account for $10 \%$ or more of the total berry. However, there are few studies on fatty acid composition of seeds of Hippophae rhamnoides L. Fatty acids (FAs) are important for human health, because many researches has proved that fatty acids play an essential role in the prevention and treatment of cancer ${ }^{12}$, cardiovascular disease ${ }^{13}$, inflammatory diseases ${ }^{14}$, retinitis pigmentosa ${ }^{15}$, chronic disease, normal health and schizophrenia etc. ${ }^{16}$. Therefore, the study of fatty acid composition is equally important to the pharmacological research of Hippophae rhamnoides $L$. and is also imperative for the quality control.

Since fatty acids showed neither natural UV absorption nor fluorescence property; thus, the detection of them at trace levels using absorptiometry is fairly difficult. The majority of fatty acids were determined by GC or GC-MS in the past years ${ }^{17,18}$. The method has its own merits, however, there are some obvious drawbacks compared to HPLC. Firstly, the main disadvantage of GC over HPLC is higher temperature during analysis procedure, which increased the risk of isomerization 
of double bonds and decreased the possibility of fraction collection for further investigation. Secondly, it has been proposed that the injection technique, especially in vaporizing injectors, is the main source of errors in quantitative $\mathrm{GC}^{19}$. Long chain polyunsaturated fatty acids were unstable during GC analysis, therefore, the accuracy was greatly affected ${ }^{20}$. Thirdly, GC could not allows fatty acid to be converted to a large number of different derivatives and the derivatization can result in some problems, such as tailing peaks and low detector sensitivity. As for the less polar compounds, these could not be easily analyzed by $\mathrm{GC}^{21}$.

Sensitive HPLC analysis of fatty acids can be achieved by derivatization of the carboxyl moiety with a suitable chromophore or fluorophore. Recently, a fluorescent probe named 2-(11H-benzo[a]carbazol-11-yl) ethyl 4-methylbenzenesulfonate (BCETS) was developed and successfully employed to carboxylic acid analysis ${ }^{22}$. In this study, the reagent was used as pre-column derivatization reagent to determinate fatty acid by HPLC fluorescent detection and the derivatives were simultaneous identified by APCI-MS. This established method exhibited excellent fluorescent sensitivity and good reproducibility. In the present study, it was applied to determinate fatty acid composition in seeds of Hippophae rhamnoides $L$. seeds from various origins of Qinghai-Tibetan Plateau and a satisfied result was obtained.

\section{EXPERIMENTAL}

The HPLC analysis was carried out using an Agilent 1100 series HPLC system equipped with an on-line-degasser, a quaternary pump, an autosampler and a thermostated column compartment. A fluorescence detector (model G1321A, Agilent, USA) was adjusted at wavelengths of 279 and 380 $\mathrm{nm}$ for excitation and emission. Chromatographic separation was achieved on a Hypersil BDS C8 column $(200 \times 4.6 \mathrm{~mm}$, $5 \mu \mathrm{m}$, Dalian Elite Analytical Instruments Co., Ltd., China). Solvent A was $5 \%$ acetonitrile in water and B was acetonitrile. The flow rate was constant at $1 \mathrm{~mL} \mathrm{~min}^{-1}$ and the column temperature was kept at $30{ }^{\circ} \mathrm{C}$. The gradient condition of mobile phase was as follows: $65-83 \% \mathrm{~B}$ from 0 to $35 \mathrm{~min}$; $83-88 \%$ B from 35 to $50 \mathrm{~min}$; 88-100 \% B from 50 to $55 \mathrm{~min}$ and then held for $5 \mathrm{~min}$. The injection volume was $10 \mu \mathrm{L}$. The column was equilibrated with the initial mobile phase for $5 \mathrm{~min}$ before the next injection.

All fatty acids used as standards were of chromatographic grade and purchased from Sigma Reagent Co. (USA). HPLC grade acetonitrile was purchasedfrom Yuwang Company, China. Dimethylformamide (DMF) potassium carbonate $\left(\mathrm{K}_{2} \mathrm{CO}_{3}\right)$, petroleum ether were of analytical grade obtained from Shanghai Chemical Reagent Co. (Shanghai, China). Water was purified on a Milli-Q system (Millipore, Bedford, MA, USA). All other reagents used were also of analytical grade unless otherwise stated. BCETS was synthesized in our laboratory.

Samples: Seeds of Hippophae rhamnoides L. were collected from DaTong county (longitude $101^{\circ} 32.843$, latitude $37^{\circ} 10.868$, elevation $2801 \mathrm{~m}$ ), HuZhu county (longitude $101^{\circ} 50.926$, latitude $36^{\circ} 57.670$, elevation $3029 \mathrm{~m}$ ), LeDu county (longitude $102^{\circ} 12.318$, latitude $36^{\circ} 19.577$, elevation
2997 m), PingAn county (longitude 101 57.494 , latitude $36^{\circ} 23.173$, elevation $2683 \mathrm{~m}$ ), XunHua county (longitude $102^{\circ} 32.184$, latitude $35^{\circ} 41.331$, elevation $2661 \mathrm{~m}$ ), HuaLong county (longitude $102^{\circ} 07.717$, latitude $36^{\circ} 03.509$, elevation $2976 \mathrm{~m}$ ), Huang Zhong county (longitude $101^{\circ} 24.426$, latitude $36^{\circ} 52.329$, elevation $2811 \mathrm{~m}$ ), MenYuan county (longitude $102^{\circ} 07.286$, latitude $37^{\circ} 12.083$, elevation $2626 \mathrm{~m}$ ), in Qinghai province in September, 2013. The fruits were dried naturally and divided into two parts of seeds and sarcocarps and then seeds were ground and sieved (32-60 mesh) for the next step test.

Preparations of standard solutions and samples: The labeling reagent solution $\left(5 \times 10^{-3} \mathrm{~mol} \mathrm{~L}^{-1}\right)$ was prepared by dissolving $20.75 \mathrm{mg}$ BCETS with $10 \mathrm{~mL}$ acetonitrile. The corresponding derivatization reagent solution of low concentration $\left(1 \times 10^{-4} \mathrm{~mol} \mathrm{~L}^{-1}\right)$ was obtained by diluting the stock solution with acetonitrile. Individual standards of fatty acids were prepared by dissolving the fatty acid in HPLC grade acetonitrile to a concentration of $1 \times 10^{-2} \mathrm{~mol} \mathrm{~L}^{-1}$. Standards of 20 mixed fatty acids $\left(1 \times 10^{-4} \mathrm{~mol} \mathrm{~L}^{-1}\right)$ were prepared by diluting the corresponding individual stock solutions with acetonitrile and mixing them up. When not in use, all reagent solutions were stored at $4{ }^{\circ} \mathrm{C}$ in a refrigerator until HPLC analysis.

The prepared samples $(0.4 \mathrm{~g})$ were added to $8 \mathrm{~mL}$ petroleum ether in a $10 \mathrm{~mL}$ volumetric flask and placed overnight. The extracted process was operated with ultrasonication for $1 \mathrm{~h}$ and the supernatant was collected. Then $4 \mathrm{~mL}$ petroleum ether was added into the flask for a second extraction. The twice supernatants were united and $1 \mathrm{~mL}$ was removed to dry under a stream of nitrogen gas for the next analysis.

Derivatization of fatty acids: $100 \mu \mathrm{L}$ standard fatty acid mixtures were added to a $2 \mathrm{~mL}$ vial containing $15 \mathrm{mg} \mathrm{K} \mathrm{CO}_{3}$ and $200 \mu \mathrm{L}$ DMF. The vial was sealed and allowed to react in a water bath at $90{ }^{\circ} \mathrm{C}$ for $0.5 \mathrm{~h}$. After the reaction was completed, the mixture was cooled to room temperature. $200 \mu \mathrm{L}$ acetonitrile was added to dilute the derivatization solution. The diluted derivatization solution $(10 \mu \mathrm{L})$ was injected directly into the chromatograph column. The derivatization procedure is shown in Fig. 1.

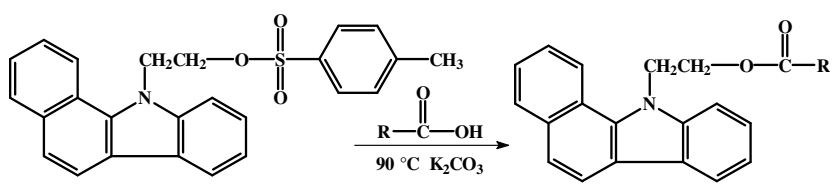

Fig. 1. Derivatization scheme of BCETS with fatty acids

MS conditions: APCI was employed to identify fatty acid derivatives. APCI conditions: nebulizer pressure, $413 \mathrm{kPa}$; dry gas temperature, $350{ }^{\circ} \mathrm{C}$; dry gas flow, $5 \mathrm{~L} \mathrm{~min}{ }^{-1}$; APCI Vap temperature, $450{ }^{\circ} \mathrm{C}$; corona current (nA), 4000 (pos); capillary voltage, $3500 \mathrm{~V}$. The positive-ion detection mode was selected for APCI ion sources.

\section{RESULTS AND DISCUSSION}

Chromatographic separation and mass spectrometry identification: To get a good command of the fatty acids that may exist in seeds of Hippophae rhamnoides L., 20 fatty acids 
were chosen in this paper; 15 of them are saturated fatty acids and the others are unsaturated fatty acids. It is difficult to separate the 20 fatty acids in a common chromatography column because unsaturated fatty acids often co-elute with saturated fatty acids containing one or two fewer carbon atoms. C18 columns showed much better effects than $\mathrm{C} 8$ columns in the aspect of fatty acid separation. However, a longer time was consumed using $\mathrm{C} 18$ column to make the separation completed (more than $120 \mathrm{~min}$ ). Therefore, C18 column was not suitable for latter experiments. Several kinds of C8 columns have been used to obtain a best separation in short time. The results indicated that Hypersil BDS C8 column in conjunction with a gradient elution could separate the 20 fatty acid derivatives within 60 min (Fig. 3A). Therefore, the Hypersil BDS C8 column was employed in this study. In addition, the mobile phase was investigated and the results showed that acetonitrilewater (5:95) (solvent A) and $100 \%$ acetonitrile (solvent B) as the mobile phases performed the sharp peak shapes in the short separation time.

Chromatographic peaks were simultaneously identified by online post-column mass spectrometry in positive mode. All molecular ions $[\mathrm{M}+\mathrm{H}]^{+}$of 20 fatty acids derivatives were listed in Table-1. The MS, MS/MS and cleavage mode for the typical C18:2 derivative were shown in Fig. 2. C18:2 derivative produced an intense molecular ion peak at $\mathrm{m} / \mathrm{z} 524$ and the specific fragment ions at 307, 261.7 and $\mathrm{m} / \mathrm{z} 243$.9. The characteristic fragment ion at $m / z 307$ was produced from the cleavage of $\mathrm{N}-\mathrm{C}$ bond of the inner structure the N-linked side chain. The specific fragment ion at $\mathrm{m} / \mathrm{z}, 243.9$ was generated from the cleavage of C-OCO bond. The peculiar fragmention at $\mathrm{m} / z$ 261.7 was from the cleavage of O-CO bond of the BECTSfatty acid derivative.

Method valuation: The method was validated for linearity, limits of detection and limit of quantification (LODs and LOQs), precision and accuracy. Linearity was estimated according to the regression of the peak area versus concentration of each fatty acid standard. All the correlation coefficients of the fatty acids were found to be greater than 0.9993, which indicated that all fatty acid standards have excellent linear responses. Limit of detection was carried out as the compound concentration that produced a signal-to-noise ratio of $3(\mathrm{~S} / \mathrm{N}=3)$, which ranged from 0.42 to $1.82 \mathrm{ngm} \mathrm{L}^{-1}$. Limit of quantification was carried out as the compound concentration that produced a signal-to-noise ratio of $10(\mathrm{~S} / \mathrm{N}$ $=10$ ), which ranged from 1.34 to $6.64 \mathrm{ngm} \mathrm{L}^{-1}$. The precision of the chromatographic method was checked three times by applying the whole procedure to sample analysis and the values of R.S.D obtained were in the range of 1.89 to $4.91 \%$. To evaluate the accuracy of the method, the recovery experiments were carried out by analyzing samples spiked the fatty acid standard mixture at levels of $2.5,5$ and $10 \mu \mathrm{mol} \mathrm{L} \mathrm{L}^{-1}$ and the results were satisfactory with the recovery range of $92.6 \%$ to $102.4 \%$. All the data of method validation were listed in Table-1.
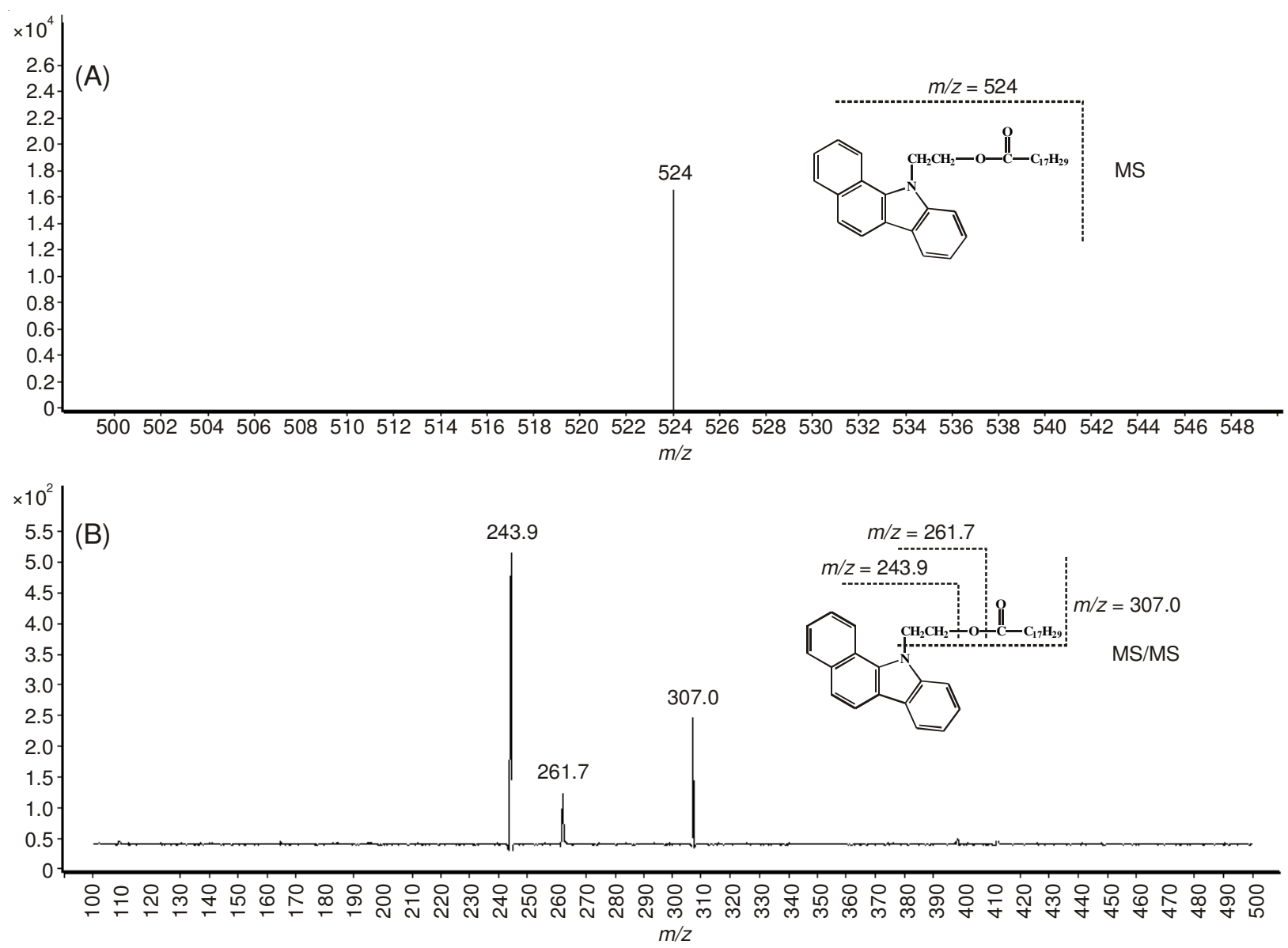

Fig. 2. MS and MS/MS spectra of representative linoleic acid (C18:2) derivative and the cleavage mode of protonated molecular ion 


\begin{tabular}{ccccccc}
\hline \multicolumn{7}{c}{ TABLE-1 $^{+}$} \\
\multicolumn{7}{c}{$\begin{array}{c}\text { MOLECULAR IONS [M+H }]^{+}, \text {r, LODs, LOQs, } \\
\text { RECOVERY AND PRECISION VALUES }\end{array}$} \\
\hline $\begin{array}{c}\text { Fatty } \\
\text { acid }\end{array}$ & {$[\mathrm{M}+\mathrm{H}]^{+}$} & $\mathrm{r}$ & $\begin{array}{c}\text { LOD }^{-1} \\
\left(\mathrm{ng} \mathrm{mL}^{-1}\right)\end{array}$ & $\begin{array}{c}\text { LOQ } \\
\left(\mathrm{ng} \mathrm{mL}^{-1}\right)\end{array}$ & $\begin{array}{c}\text { Recovery } \\
(\%)\end{array}$ & $\begin{array}{c}\text { RSD } \\
(\%)\end{array}$ \\
\hline C10 & 416.3 & 0.9999 & 0.53 & 1.61 & 98.6 & 2.44 \\
C11 & 430.1 & 0.9995 & 0.42 & 1.34 & 99.3 & 3.11 \\
C12 & 444.3 & 0.9999 & 0.61 & 1.89 & 95.5 & 1.89 \\
C13 & 458.3 & 0.9996 & 0.59 & 1.67 & 101.9 & 3.05 \\
C18:3 & 521.9 & 0.9997 & 0.67 & 1.91 & 99.8 & 1.98 \\
C14 & 472.3 & 0.9998 & 0.55 & 1.71 & 97.9 & 2.31 \\
C16:1 & 498.2 & 0.9996 & 0.73 & 2.32 & 93.5 & 2.43 \\
C18:2 & 523.9 & 0.9993 & 0.76 & 2.54 & 97.3 & 3.50 \\
C15 & 486.3 & 0.9999 & 0.81 & 2.61 & 98.6 & 2.79 \\
C16 & 500.2 & 0.9995 & 0.87 & 2.73 & 96.5 & 3.38 \\
C18:1 & 521.9 & 0.9994 & 0.92 & 2.98 & 99.7 & 4.11 \\
C17 & 514.4 & 0.9995 & 0.88 & 2.82 & 92.6 & 4.25 \\
C18 & 527.6 & 0.9997 & 0.99 & 3.21 & 94.8 & 3.77 \\
C20:1 & 553.9 & 0.9997 & 1.12 & 3.54 & 99.3 & 3.90 \\
C19 & 542.4 & 0.9999 & 1.23 & 3.73 & 102.4 & 2.98 \\
C20 & 556.3 & 0.9998 & 1.41 & 4.15 & 94.2 & 3.33 \\
C21 & 570.3 & 0.9997 & 1.51 & 4.74 & 93.5 & 4.11 \\
C22 & 584.1 & 0.9998 & 1.62 & 4.95 & 96.1 & 4.32 \\
C23 & 598.4 & 0.9996 & 1.76 & 5.32 & 93.5 & 3.89 \\
C24 & 612.3 & 0.9995 & 1.82 & 6.64 & 94.8 & 4.91 \\
\hline & & & & & & \\
\hline
\end{tabular}

Analysis of fatty acids in seeds of Hippophae rhamnoides

L.: The proposed method was successfully employed to the analysis of fatty acids in seeds of Hippophae rhamnoides $L$. Standard solution was injected after every 5 samples to make up the possible deviation in retention times. Fig. 3 shows a representative chromatogram of fatty acid standard solution (A) and a chromatogram of fatty acids in the seeds of Hippophae rhamnoides $L$. (B). The fatty acid content of seeds of Hippophae rhamnoides L. from various origins were summarized in Table-2. The results showed the main unsaturated fatty acids existed in seeds of Hippophae rhamnoides $L$. were linoleic acid (C18:2), oleic acid (C18:1) and linolenic acid (C18:3). Saturated fatty acids were mainly C16 (hexadecanoic acid), C18 (octadecanoic acid). Besides, small amounts of C12 (dodecanoic acid), C13 (tridecanoic acid), C14 (myristic acid), C15 (pentadecanoic acid), C20 (arachidic acid), C22 (behenic acid) and $\mathrm{C} 24$ (tetracosanoic acid) were also found to be existed in seeds of Hippophae rhamnoides L. As can be seen in Table-2, unsaturated fatty acids levels were higher than that of unsaturated fatty acids, the contents of which occupied $72 \%$ or more of total fatty acids (TFAs). The most unsaturated fatty acid was linoleic acid (C18:2) with the highest and lowest amount
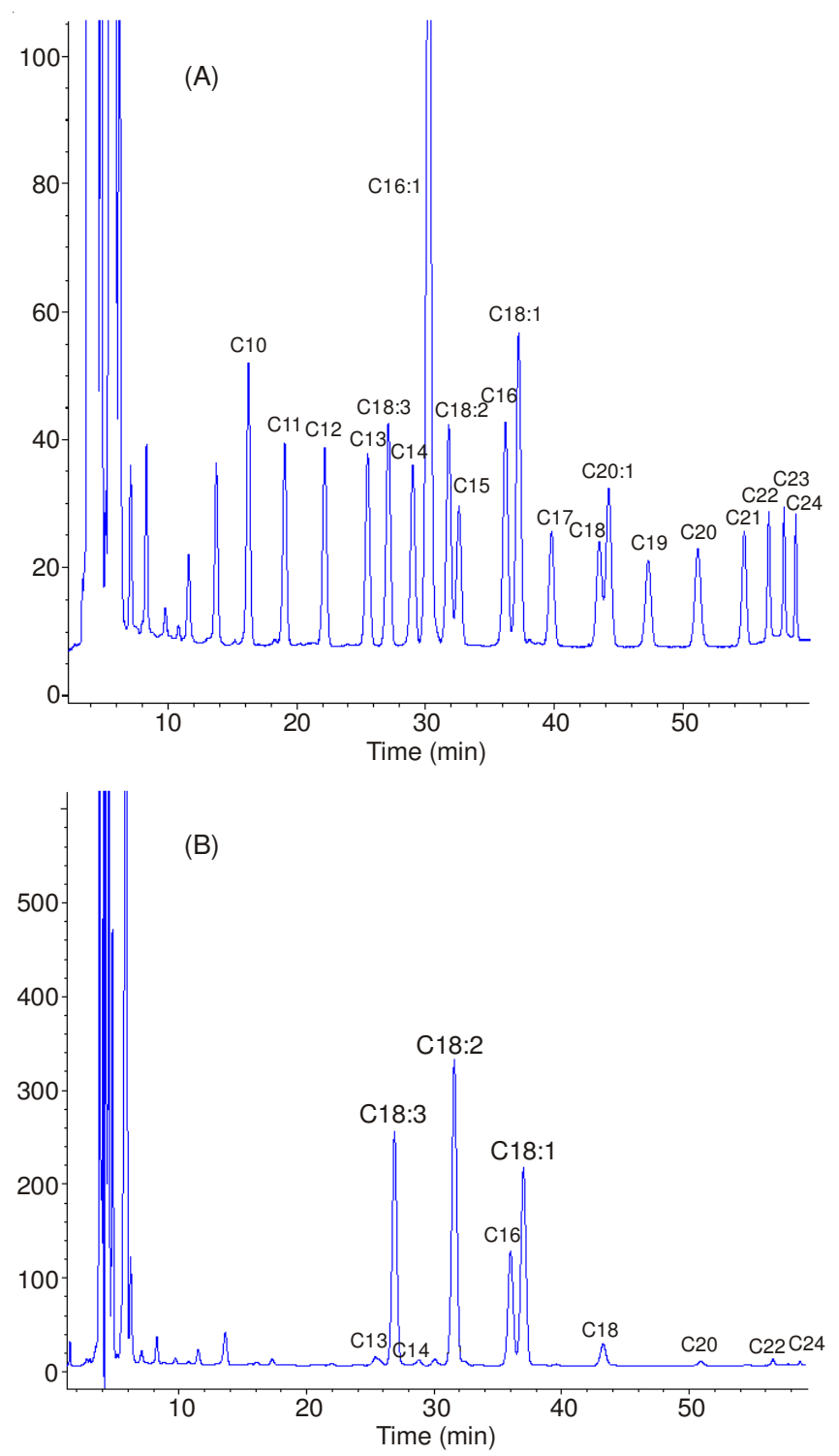

Fig. 3. Chromatograms of fatty acids derivatives from (A) 20 fatty acid standards and (B) seeds of seeds of Hippophae rhamnoides L. Peak labels: C10 (decoic acid); C11 (undecanoic acid); C12 (dodecanoic acid); C13 (tridecanoic acid); C18:3 (8,11,14-octadecatrienoic acid); C14 (myristic acid); C16:1 (palmitoleic acid); C18:2 (9,12octadecadienoic acid); C15 (pentadecanoic acid); C16 (hexadecanoic acid); C18:1 (12-octadecenoic acid); C17 (heptadecanoic acid); C18 (octadecanoic acid); C20:1 (11eicosenoic acid); C19 (nonadecanoic acid); C20 (arachidic acid); C21 (heneicosoic acid); C22 (docosanoic acid); C23 (tricosanoic acid); C24 (tetracosanoic acid)

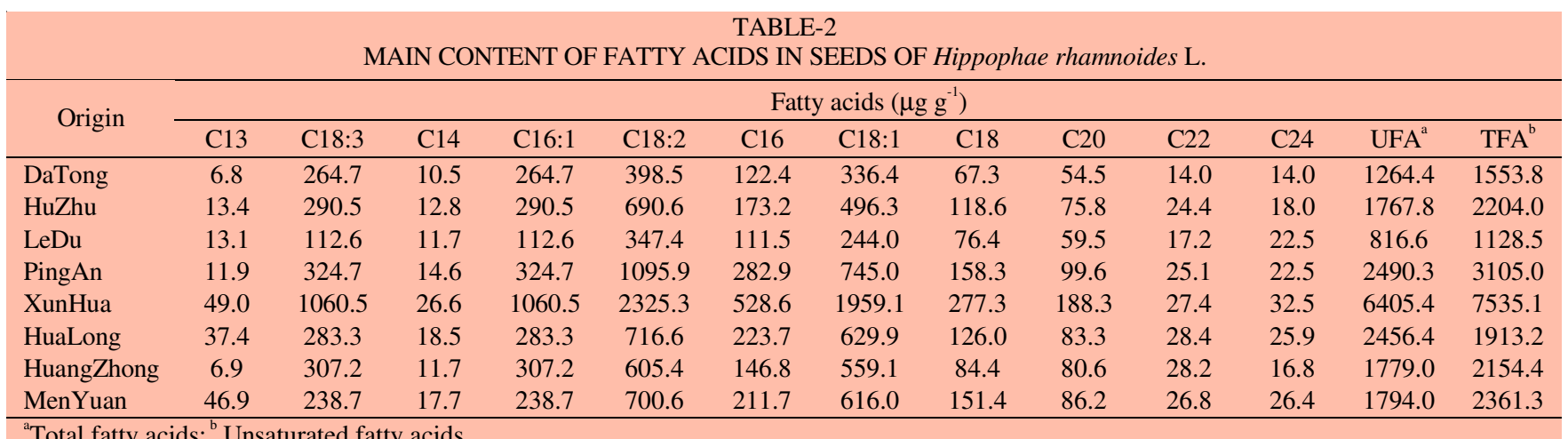


of $2325.3 \mu \mathrm{g} \mathrm{g}^{-1}$ (XunHua) and $347.4 \mu \mathrm{g} \mathrm{g}^{-1}$ (LeDu), respectively. It was found that palmitic acid (C16) was the most abundant unsaturated fatty acid in all seeds of Hippophae rhamnoides $L$. from various origins. The highest and lowest concentrations were $528.6 \mu \mathrm{g} \mathrm{g}^{-1}$ (XunHua) and $111.5 \mu \mathrm{g} \mathrm{g}^{-1}$ $(\mathrm{LeDu})$, respectively. The highest and lowest contents of total fatty acids were also found from XunHua and LeDu regions and the total fatty acid contents of the corresponding samples were $7535.1 \mu \mathrm{g} \mathrm{g}^{-1}$ and $1128.5 \mu \mathrm{g} \mathrm{g}^{-1}$, respectively. In addition, whatever total fatty acids, unsaturated fatty acids or each fatty acid, there were also significant differences among various origins (Table-2). Beyond these, C10 (decoic acid), C11 (undecanoic acid), C12 (dodecanoic acid), C17 (heptadecanoic acid), C20:1 (11- eicosenoic acid), C19 (nonadecanoic acid), C21 (heneicosoic acid), C23 (tricosanoic acid) were not detected using the proposed method.

\section{Conclusion}

A selective and sensitive method using BCETS as precolumn derivatization reagent for the analysis of fatty acids has been proposed and validated in the present work. With the optimum chromatographic separation conditions, 20 fatty acids were separated with good separation degree. This proposed method was successfully applied to the qualitative and quantitative analysis of fatty acids existed in seeds of Hippophae rhamnoides $L$. The results indicated that the contents of fatty acids in seeds of Hippophae rhamnoides $L$. of various origins were significantly different and the samples from XunHua and LeDu contain the highest and lowest fatty acids. But, the main fatty acids containing palmitic (C16), stearic (C18), oleic (C18:1), linoleic (C18:2) and linolenic (C18:3) acids in the seeds of seeds of Hippophae rhamnoides $L$. from different origins were similar. It is believed that this work would facilitate the use of seeds of Hippophae rhamnoides $L$. in pharmaceutical application and contribute to the quality control.

\section{ACKNOWLEDGEMENTS}

The work was supported by China spark program (No. 2011GA870007).

\section{REFERENCES}

1. A. Rousi, Ann. Bot. Fenn., 8, 177 (1971).

2. L. Rongsen, Kathmandu: International Centre for Integrated Mountain Development, ISBN 701215708, p. 62 (1992).

3. H. Suleyman, K. Gumustekin, S. Taysi, S. Keles, N. Oztasan, O. Aktas, K. Altinkaynak, H. Timur, F. Akcay, S. Akar, S. Dane and M. Gul, Biol. Pharm. Bull., 25, 1133 (2002).

4. S. Geetha, M. Sai Ram, V. Singh, G. Ilavazhagan and R.C. Sawhney, J. Ethnopharmacol., 79, 373 (2002).

5. J. Purushothaman, G. Suryakumar, D. Shukla, A.S. Malhotra, H. Kasiganesan, R. Kumar, R.C. Sawhney and A. Chami, Brain Res. Bull., 77, 246 (2008).

6. H. Süleyman, L.Ö. Demirezer, M.E. Büyükokuroglu,M.F. Akcay, A. Gepdiremen, Z.N. Banoglu and F. Göçer, Phytother. Res., 15, 625 (2001).

7. L. Ganju, Y. Padwad, R. Singh, D. Karan, S. Chanda, M.K. Chopra, P. Bhatnagar, R. Kashyap and R.C. Sawhney, Int. Immunopharmacol., 5, 1675 (2005).

8. B.S. Teng, Y.H. Lu, Z.T. Wang, X.Y. Tao and D.Z. Wei, Pharmacol. Res., 54, 186 (2006).

9. A. Ranjith, K.S. Kumar, V.V. Venugopalan, C. Arumughan, R.C. Sawhney and V. Singh, J. Am. Oil Chem. Soc., 83, 359 (2006).

10. A. Raffo, F. Paoletti and M. Antonelli, Eur. Food Res. Technol., 219, 360 (2004).

11. X. Pang, J. Zhao, W. Zhang, X. Zhuang, J. Wang, R. Xu, Z. Xu and W. Qu, J. Ethnopharmacol., 117, 325 (2008).

12. R. Colomer, J.M. Moreno-Nogueira, P.P. García-Luna, P. García-Peris, A. García-de-Lorenzo, A. Zarazaga, L. Quecedo, J. del Llano, L. Usán and C. Casimiro, Br. J. Nutr., 97, 823 (2007).

13. P.M. Kris-Etherton, W.S. Harris and L.J. Appel, Arterioscler. Thromb. Vasc. Biol., 23, e20 (2003).

14. P.C. Calder, Am. J. Clin. Nutr., 83, S1505 (2006).

15. W.G. Hodge, D. Barnes, H.M. Schachter, Y.I. Pan, E.C. Lowcock, L. Zhang, M. Sampson, A. Morrison, K. Tran, M. Miguelez and G. Lewin, Can. J. Ophthalmol., 41, 481 (2006).

16. A.P. Simopoulos, Am. J. Clin. Nutr., 70, 560s (1999).

17. E. Pusvaskiene, B. Januskevic, A. Prichodko and V. Vickackaite, Chromatographia, 69, 271 (2009).

18. J. Gamazo-Vázquez, M.S. Garcia-Falcón and J. Simal-Gándara, Food Contr., 14, 463 (2003).

19. L. Silva, A. Cachada, R. Pereira, A.C. Freitas, T.A.P. Rocha-Santos, T.S.L. Panteleitchouk, M.E. Pereira and A.C. Duarte, Talanta, 85, 222 (2011).

20. M. Schreiner, J. Chromatogr. A, 1095, 126 (2005).

21. D. Perret, A. Gentili, S. Marchese, M. Sergi and L. Caporossi, Rapid Commun. Mass Spectrom., 18, 1989 (2004).

22. G. Li, C. Song, J. You, X. Zhang and G. Chen, J. Am. Oil Chem. Soc., 89, 585 (2012). 